\title{
RESERVA PENÍNSULA DE SAN JULIÁN: ESTUDIOS ARQUEOLÓGICOS DISTRIBUCIONALES EN UNA PARTICULAR GEOFORMA MARINA
}

\author{
RAFAEL S. PAUNERO Y FABIANA SKARBUN
}

\begin{abstract}
RESUMEN
Presentamos los resultados de los trabajos de arqueología distribucional realizados en la península de San Julián, considerada como una geoforma diferente dentro del paisaje de costa, que sugiere una manera particular de utilización por parte de los grupos humanos. En este sentido, nuestros objetivos se orientan a conocer el rol que ocupó la península a partir del establecimiento de la línea de costa actual, dentro de las diferentes estrategias de explotación y los probables patrones espaciales de usos implementados para los diferentes ambientes de la región.

Las tareas de campo consistieron en prospecciones realizadas en toda la península, siguiendo un diseño metodológico particular planteado en dos etapas. Las distribuciones y densidades de artefactos líticos registradas permitieron definir diferente intensidad de uso según la unidad de paisaje, identificar sitios y ubicar sectores viables de excavación. Los resultados generados, permiten visualizar, en mapa, la distribución de los elementos en superficie que hacen al uso del espacio de costa e interior por parte de los grupos humanos.
\end{abstract}

PALABRAS CLAVE: península, distribución, densidades, usos, espacios.

\section{RESERVA PENÍNSULA DE SAN JULIÁN: DISTRIBUTIONAL ARCHAEOLOGICAL STUDIES IN A PARTICULAR COASTAL GEOMORPHOLOGY}

\begin{abstract}
We present the results of our studies on distributional archaeology in San Julián Peninsula, considered a particular geomorphology in the coastal landscape, which suggests it was used in a special way by human groups. We aim to know which role played the peninsula in the different strategies of exploitation and the probable special use patterns implemented for the different environments in the region, from the moment the present coast line was established.
\end{abstract}


We surveyed the whole peninsula through a methodological design which consisted on two stages. The distribution and density of lithic artifacts were recorded. They allowed us to define different use intensities according to landscape units, identify sites and recognize possible digging areas. The results make visible on a map the distribution of surface elements related to the use of coastal and interior spaces by human groups.

KEY WORDS: peninsula, distribution, densities, uses, spaces.

\section{INTRODUCCIÓN}

Los estudios arqueológicos de la costa son necesarios para relacionar los diferentes contextos arqueológicos y tratar de integrar en modelos regionales los conocimientos generados por las diferentes líneas de investigación en curso. Se trata de considerar los diferentes ambientes en referencia a los grupos cazadores recolectores que los habitaron, en un amplio marco temporal, con miras a la resolución de las problemáticas arqueológicas planteadas a partir de la relación entre espacios geográficos distintos. Este trabajo se encuadra dentro del proyecto "Arqueología y Comunicación en la Reserva Península de San Julián, provincia de Santa Cruz", que tiene como fin conocer las distintas formas de ocupación humana y el uso que tuvo la península, identificar patrones de ocupación del espacio y explotación de recursos económicos, conocer la distribución, densidad y características tecnológicas de los artefactos, generar información ambiental y paleoambiental, identificar variaciones del paisaje temporales y espaciales, evaluar los procesos de formación del registro arqueológico y ponderar la visibilidad arqueológica.

La península es considerada una particular geoforma de costa, dentro de las diferentes estrategias de explotación de ambientes implementadas por los grupos humanos que ocuparon el actual territorio de Santa Cruz. Desde este punto de vista, su estudio complementa las investigaciones arqueológicas que venimos desarrollando desde hace varios años en la provincia, permitiendo integrar al proyecto los contenidos arqueológicos producidos por otras investigaciones en curso referidas a meseta, costa y zonas intermedias. Se trata de inferir patrones especiales de uso de este ambiente costero, incorporando la información a los modelos de carácter regional. En este trabajo particular nos ocupa específicamente explorar patrones de ocupación del espacio a partir del estudio de la distribución y densidad de artefactos utilizando los mapas de distribución.

\section{DESCRIPCIÓN DEL ÁREA DE ESTUDIO}

La Reserva Península de San Julián (49ํㅜㅇ

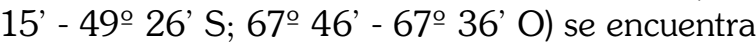
dentro del departamento de Magallanes, al Sureste de la ciudad de Puerto San Julián, enmarcando a la bahía del mismo nombre. Comprende una superficie total de 10.400 hectáreas y está conformada por dos sectores: el área interior continental y la península propiamente dicha, esta última, motivo de este informe, es una angosta extensión de tierra cercada por el mar que posee dos costas diferentes, una hacia el mar abierto y otra hacia la bahía, separadas por una relativa corta distancia. Esta península es de $18 \mathrm{Km}$. de largo y de 3,5 a $5 \mathrm{Km}$. de ancho y se encuentra unida al continente por una zona de acceso y estrangulamiento (Figura 1). Podríamos decir que la primera observación científica corresponde a Darwin, quien en Punta Asconapé en el extremo norte de la península, halló y determinó en un contexto fosilífero, restos óseos del gran mamífero extinguido, Macrauchenia patachonica, incluso describiendo el perfil de la terraza norte (Darwin 1886).

Diferentes estudios se han realizado desde el punto de vista geológico y geomorfológico de la costa patagónica (Feruglio 1950; Codignotto 1990; 1996, entre otros), en particular el sector que nos ocupa fue mapeado por Panza e Irigoyen (1994). De acuerdo a estos antecedentes, podemos decir que la costa de la provincia de Santa Cruz es el resultado de un proceso de emersión, con ascensos discontinuos, acompañados por eventos de acreción (Pereyra et al 2002:345-346). Se registran en las cercanías de la Bahía San Julián, tres niveles de acreción marina, los niveles de depositación de cordones intermedios, ocurridos alrededor de 30.000 años AP modificaron la línea de costa, que anteriormente era más irre- 


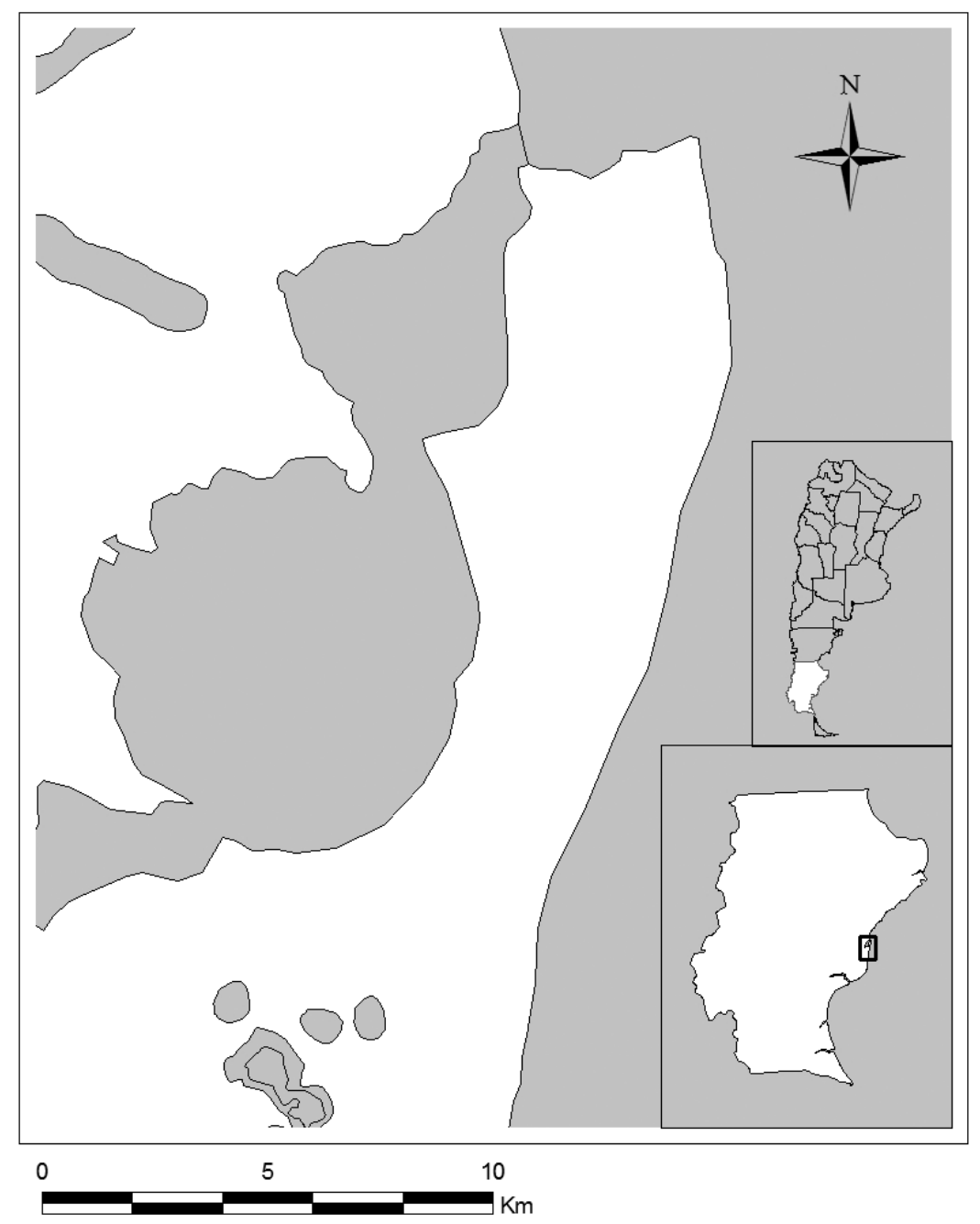

Fig. 1. Ubicación de Península de San Julián.

gular. En el sector interior de la reserva, fuera de la península y a $4 \mathrm{Km}$. de la costa atlántica, registramos las terrazas y paleoplayas correspondientes a este nivel, en lo que hoy es un bajo salitroso. Si bien es necesario ajustar y desarrollar la geomorfología y geología particular del área, podemos decir que aproximadamente entre 6.000 y 7.000 años AP, a partir del desarrollo del tercer nivel de acreción de cordones litorales, el ambiente marino de la bahía se hizo más restringido y la península alcanza dimensiones y características bastante similares a las actuales (Panza e Irigoyen 1994; Pereyra et al 2002).

Consideramos pertinente hacer aquí una breve descripción de la utilización que tuvo este lugar a partir de la ocupación de europeos y sus descendientes desde fines del siglo XIX, por ser útil para ponderar los procesos de alteración de sitios y la construcción del paisaje tal como lo percibimos en la actualidad. De acuerdo a datos propios, a informantes varios y principalmente al informe de Manessi (2007), la ocupación por parte de ganaderos comienza en la última década del siglo XIX. En el año 1899 pasó a formar parte del espacio reservado para fundación del pueblo y su ensanche en una futura colonización. Su utilización para cría de ganado ovino finaliza en el año 1986, momento en que fue creada la Reserva Provincial Península de San Julián por Ley Provincial № 1821/86 (Manessi 2007). Es importante señalar que en la actualidad está reestablecida la población de guanacos en un número aproximado a 200 
individuos que utilizan la reserva como campos de parición y cría. Por último cabe indicar que desde hace aproximadamente veinte años, la reserva es utilizada por la comunidad de Puerto San Julián principalmente como lugar de pesca y extracción de áridos en algunos sectores.

\section{ANTECEDENTES DE ESTUDIOS DE COSTA EN SANTA CRUZ}

Los estudios arqueológicos de la costa de Santa Cruz, evidencian un registro de ocupaciones costeras a partir de la trasgresión marina del Holoceno medio, hacia los 6.000/7.000 años AP y con el establecimiento del nivel del mar actual. Esta profundidad temporal se vincula con la evolución geomorfológica del contorno costero y a las fluctuaciones ocurridas durante el Holoceno. Para la Costa Norte de Santa Cruz los fechados datan desde ca. 6.000 años (Castro y Moreno 1998; Zubimendi et al. 2005; Zubimendi 2010), sin embargo el registro de ocupaciones datadas es mayor y más redundante en el Holoceno tardío, indicando que la costa estuvo definitivamente incorporada al territorio de las sociedades cazadoras recolectoras durante este momento (Castro et al. 2003). Los estudios proponen que este amplio litoral fue intensamente utilizado por cazadores recolectores que consumían preferentemente pinnípedos, cormoranes y moluscos (Zubimendi et al. 2005). Para este sector se prepuso un modelo de ocupación estacional donde la costa se ocuparía durante períodos correspondientes a invierno, primavera y comienzo del verano, al menos en el Holoceno tardío, con una tendencia a la diversificación económica, utilizando ambientes especiales y recursos marinos, Por otra parte, la forma de ocupación habría variado según las características geomorfológicas y la disponibilidad diferencial de recursos (Castro et al. 2008). En este sentido se reconocieron tres sectores diferentes clasificados según el número y el tamaño de los sitios: sectores de baja, media y alta densidad de uso (Castro et al. 2003; Moreno 2003). La distribución de los concheros y de los chenques indicaría un uso estructurado no eventual del espacio costero (Castro y Moreno 2000). Según los estudios de Arrigoni y otros (2008) para los sectores litorales del Golfo San Jorge en la costa existieron grupos de cazadores recolectores, posiblemente conectados culturalmente con el in- terior, pero con economías diferenciadas, producto de la explotación de dos franjas ecológicamente complementarias, agua y tierra, que posiblemente les proporcionaban mayores beneficios económicos, debido a la diversidad de recursos marinos y terrestres incorporados a la dieta.

Al sur de la Península de San Julián, en el Parque Nacional Monte León, las ocupaciones costeras datan de ca. 5.100 AP y ca. 4.100 años AP (Caracotche et al. 2008). Los estudios muestran un aprovechamiento de recursos locales e inmediatamente disponibles, y en menor proporción no disponibles localmente. Asimismo, a partir de la identificación predominante de las primeras etapas de la secuencia de reducción lítica, los investigadores del área sugirieron que debió existir algún tipo de uso complementario de la zona con otras áreas vecinas, que estarían ubicadas en ambientes distintos. De esta manera, Monte León estaría dentro del marco supra-regional de la circulación de materias primas (Caracotche et al. 2005; Borrero et al. 2008). Caracotche et al. (2008) determinaron el consumo de recursos costeros, mamíferos marinos y moluscos, aunque los artefactos líticos no muestran un uso claro para su explotación. Las evidencias sustentan la utilización de los espacios interiores como lugar de tránsito, probablemente relacionado con la circulación de poblaciones humanas y aprovechamiento de las materias primas disponibles (Borrero et al. 2008). El sector de Punta Bustamante posee diversos microambientes con accesibilidad a una variedad de recursos siendo la especie más aprovechada el guanaco, mientras que los recursos marinos fueron explotados de manera complementaria, en un marco de actividades de sociedades de cazadores-recolectores móviles que frecuentaban emplazamientos específicos (Mansur 2008).

A partir de los antecedentes y a las características particulares de la península, considerada como una geoforma diferente dentro del paisaje de costa, planteamos como hipótesis de trabajo que desde que la misma tomó su forma actual, poseyó un localización estratégica para el manejo de recursos terrestres y marinos, permitiendo una manera particular de utilización por parte de los grupos humanos durante el Holoceno tardío, con patrones especiales de uso, particularmente control de manadas de guanacos para utilización y consumo, mediante campamentos en su acceso y recolección 
complementaria de mejillones. Existirían diferencias en los patrones de uso de la península relacionados a la distintas geoformas, con una mayor intensidad de ocupación en sectores vinculados con distintos recursos económicos, principalmente faunísticos e hídricos.

\section{METODOLOGÍA}

Este análisis se ha llevado a cabo desde una perspectiva distribucional, en función de obtener información sobre los patrones de distribución de restos arqueológicos en una gran extensión areal a partir de una estrategia poco intrusiva (Borrero et al.1992; Belardi 2003; Castro et al. 2003; Moreno 2003; Zubimendi 2010), que nos permita definir áreas de ocupación, intensidad de uso y posible funcionalidad de las mismas a lo largo del tiempo.

Desde esta perspectiva consideramos el registro arqueológico como el producto de la reiterada modificación del espacio en función de la continua práctica social diferencial a través del espacio y del tiempo. El registro arqueológico es continuo y su estructura puede ser descripta en términos de la variación en la densidad artefactual a lo largo del paisaje (Foley 1981; Mena 1988-89). La simple agregación de artefactos no implica directamente un proceso específico en el pasado (Binford 1992). El registro arqueológico no puede entenderse solo como el producto directo del uso pasado del espacio, sino como producto de una amplia serie de eventos de depositación históricamente relacionados que dejaron conjuntos de artefactos de densidad variable. Los materiales se agregan, remueven y reacomodan continuamente en el registro arqueológico (Dunnell 1992). Los procesos naturales de formación de sitios, como la erosión, depósitos aluviales o eólicos interactúan con los procesos culturales y modifican las locaciones (Rossignol 1992). La unidad de observación básica son los artefactos (Binford 1992; Dunnell 1992; Belardi 2003), esto permite considerar la relación espacial existente entre los artefactos. Realizamos prospecciones de escala regional, por muestreos sistemáticos probabilísticos (Belardi 2003; Castro et al. 2003).

La metodología distribucional utilizada, que se inicia con el planteo del problema a partir de la marcha de nuestros propios proyectos y de la consulta bibliográfica, cuenta con una primera etapa, previa a la elaboración del diseño de muestreo sistemático y constituida, en este caso, por los siguientes pasos:

1. Análisis de imágenes satelitales, mapas, cartas topográficas y geológicas de la región. Consulta con informantes locales.

2. Definición de unidades analíticas a partir de información geomorfológica, ambiental, ecológica y de la estructuración de recursos. El interior fue definido como una sola unidad analítica, destacando dentro de ella tres segmentos particulares: zona de acceso y estrangulamiento, zona de pequeños bajos con lagunas temporarias y el extremo norte. La costa fue dividida en tres unidades analíticas: costa norte, costa este y costa oeste (Fig. 2).

3. Recorridos iniciales: fueron dirigidos a reconocer previamente toda la zona en estudio, sirvieron para localizar puntos paisajísticos importantes, identificar ambientes particulares y registrar las características de las concentraciones de artefactos (Fig. 3).

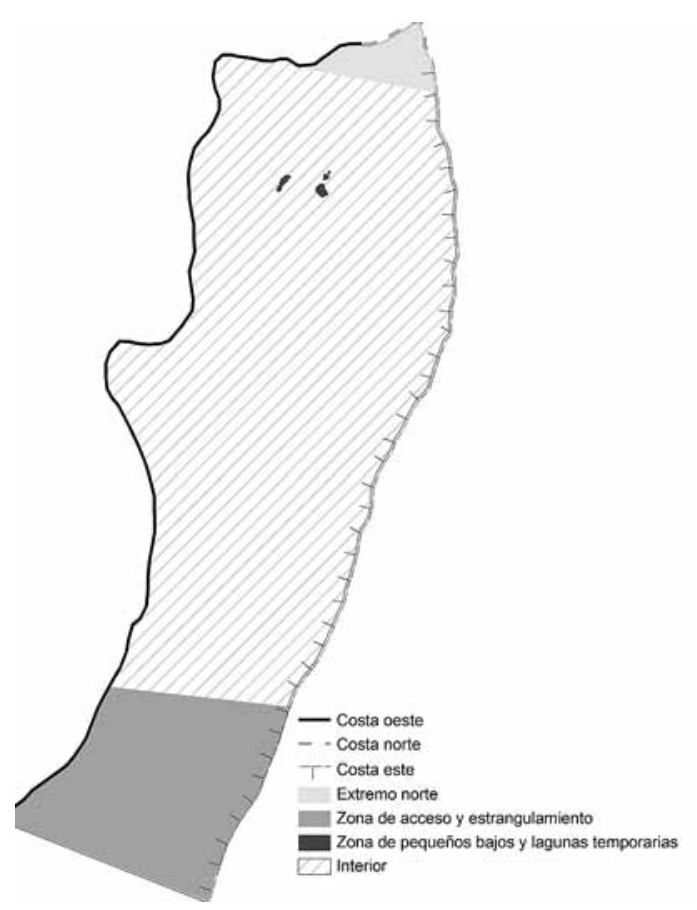

Fig. 2. Unidades analíticas. 
4. Transectas iniciales: se realizaron dos transectas iniciales, el punto inicial de ambas se encuentra al sur de la península, sobre la costa de la bahía. La primera fue realizada recorriendo $5 \mathrm{Km}$. hacia el Norte siguiendo la línea de costa. La segunda en línea recta hacia la costa atlántica, uniendo ambas costas en la zona de estrangulamiento. La realización de estas transectas permitió reconocer concentraciones de artefactos, entre ellas el sitio El Rincón ubicado sobre la transecta de la costa (Fig. 3).

A partir de los resultados obtenidos en esta primera etapa se diseñó un muestreo sistemático probabilístico de amplia escala y alta intensidad relativa, cuyo propósito fue obtener información sobre densidad y distribución artefactual, visibilidad arqueológica, ambientes y recursos.

Se definieron 34 transectas de 5 andariveles cada una, separados por $10 \mathrm{~m}$, en los que cada pros-

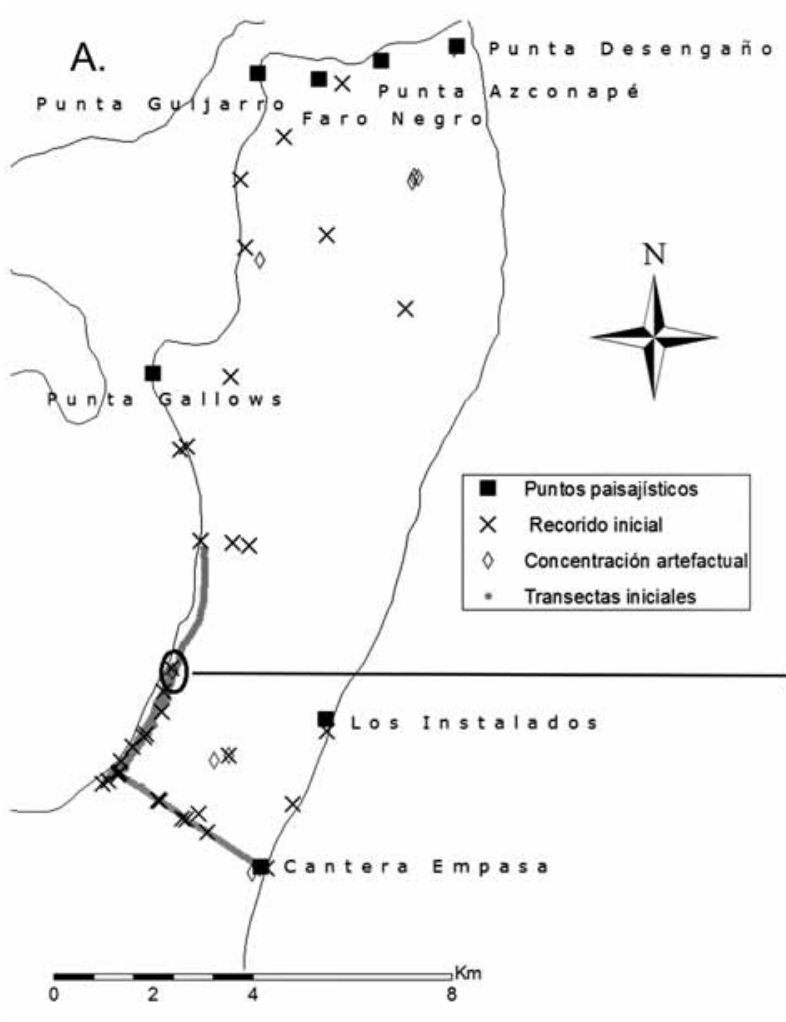

pectador caminó siguiendo la línea y cubriendo un ancho visual de $8 \mathrm{~m}$, la orientación y el trayecto se efectuó con brújula y GPS (Figura 4). Se realizaron 17 transectas interiores, con orientación este-oeste, rectas, alineadas en función de los paralelos; y 17 costeras, de $1 \mathrm{Km}$. de largo con su lado mayor equidistante a la línea costera; Cada una fue subdividida en segmentos de $50 \mathrm{~m}$ en los cuales fueron relevados todos los materiales arqueológicos identificados, los rasgos actuales y la visibilidad. El total de lo prospectado por persona fue de $102 \mathrm{Km}$. Es decir, sumando los cinco andariveles la distancia total del recorrido ha sido de aproximadamente $510 \mathrm{Km}$.

La información obtenida de cada transecta fue ingresada en una base de datos espacial de información arqueológica perteneciente a programas de sistemas de información geográfica (SIG) a partir de la cual se construyeron mapas de distribución y de densidad de artefactos líticos; también se obtuvo una medida de la densidad artefactual y se localizaron los sectores de mayor concentración. En primer lugar se analizó esta medida por transectas completas y en segundo lugar dentro de cada una, considerando la densidad por segmento de $50 \mathrm{~m}$. Luego se construyó un modelo de densidad artefactual utilizando el programa surface e interpolando por el método kriging (Izeta 2008; Bevan y Conolly 2009). La densidad arqueológica se definió sobre la base de la cantidad artefactos por unidad de muestreo. Estos muestreos sirvieron para analizar los patrones de distribución artefactual en relación a las cuatro unidades analíticas planteadas.

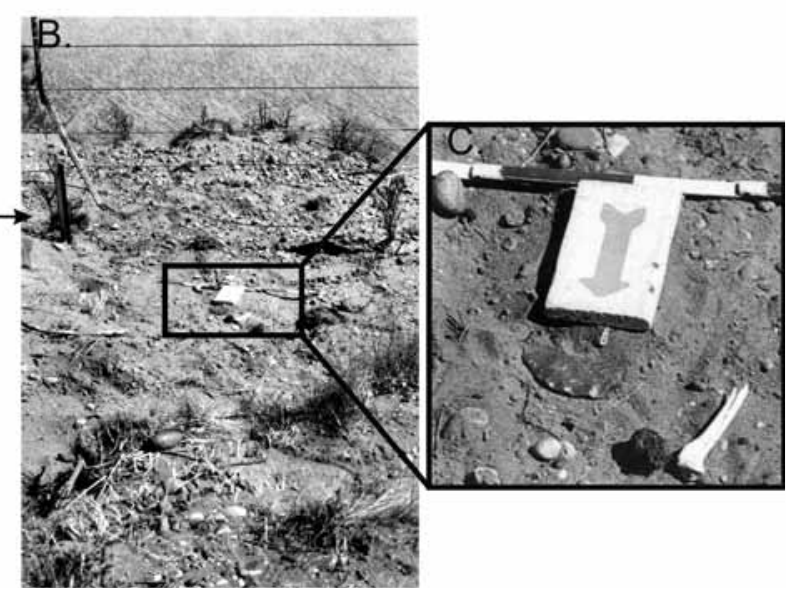

Fig. 3. A) recorridos y transectas iniciales. B) materiales arqueológicos del sitio El Rincón. C) Detalle de bifaz sitio El Rincón. 


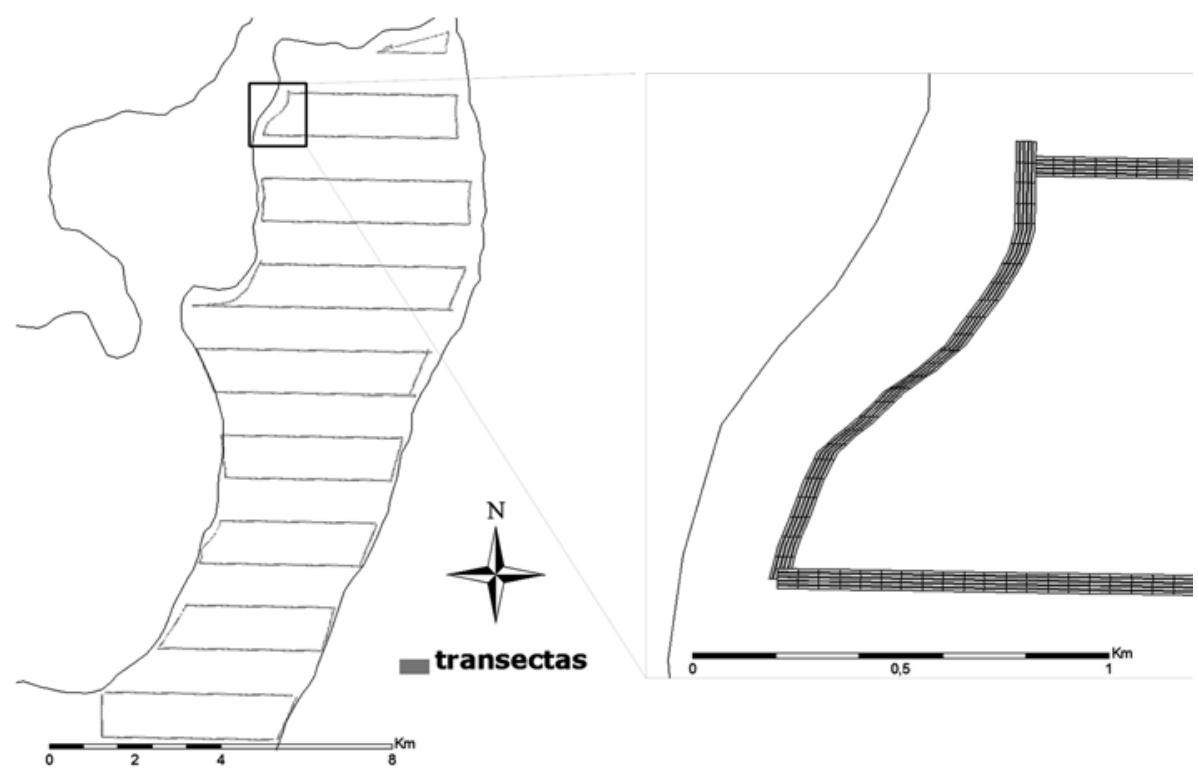

Fig. 4. Diseño del muestreo, detalle de transecta.

\section{RESULTADOS}

Las prospecciones realizadas permitieron observar el 4,342\% de la superficie total de la península (Tabla 1). La cantidad total de artefactos líticos identificada fue 2.052, su densidad por $\mathrm{m}^{2}$ es 0,000576 . Dentro de esta totalidad podemos observar que las áreas con uno o ningún artefacto lítico por $\mathrm{m}^{2}$ abarcan una superficie de 2.528.772,625 $\mathrm{m}^{2}$, que representa el 70,995 \% de la superficie total observada. Mientras que las áreas que contienen más de un artefacto lítico por $\mathrm{m}^{2}$ suman una superficie

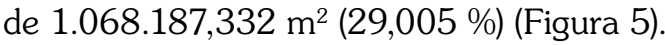

Total $361.903,71 \mathrm{~m}^{2}$

Mayor a 1; 1.068.187,33 $\mathrm{m}^{2}$;

$30 \%$

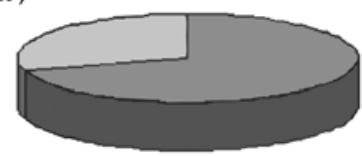

0 y 1 ;

$2.528 .772,63 \mathrm{~m}^{2}$;

$70 \%$

Fig. 5. Relación entre superficie observada y cantidad de artefactos.
Tabla 1.

\begin{tabular}{|l|r|}
\hline Superficie total & \multicolumn{1}{c|}{$\mathrm{m}^{2}$} \\
\hline Reserva Península de San Julián & $104.000 .000,000$ \\
\hline Península de San Julián & $82.033 .256,865$ \\
\hline Observada & $3.561 .903,706$ \\
\hline
\end{tabular}

En el sector costero (Fig. 6) se muestreó un área de 742.107,252 $\mathrm{m}^{2}$ donde se identificaron 584 artefactos líticos, que representan una densidad artefactual por $\mathrm{m}^{2}\left(\mathrm{art} / \mathrm{m}^{2}\right)$ de 0,000787 . La costa Este tiene una densidad de $0,000318 \mathrm{art} / \mathrm{m}^{2}$, siendo la transecta C13 la más densa con 0,00096 art/ $\mathrm{m}^{2}$ y la C5 la de menor densidad con 0,000026 $\mathrm{art} / \mathrm{m}^{2}$. La costa Oeste tiene una densidad de $0,001261 \mathrm{art} / \mathrm{m}^{2}$, la más alta se encuentra en la transecta C8 con 0,005487 art $/ \mathrm{m}^{2}$ - esta es la más alta registrada en todas las transectas - y la menor densidad artefactual fue identificada en la transecta C16, ubicada al norte de la península, con $0,000047 \mathrm{art} / \mathrm{m}^{2}$. Por último, la transecta norte posee $0,000537 \mathrm{art} / \mathrm{m}^{2}$.

El área muestreada en el interior (Fig. 7) es de $2.872 .947,017 \mathrm{~m}^{2}$, en ella se identificaron 1.468 artefactos, que representan una densidad de $0,000511 \mathrm{art} / \mathrm{m}^{2}$. La transecta 15 es la que posee mayor densidad artefactual $\left(0,003773 \mathrm{art} / \mathrm{m}^{2}\right)$ y la transecta 7 es la de menor $\left(0,000058 \mathrm{art} / \mathrm{m}^{2}\right)$. 


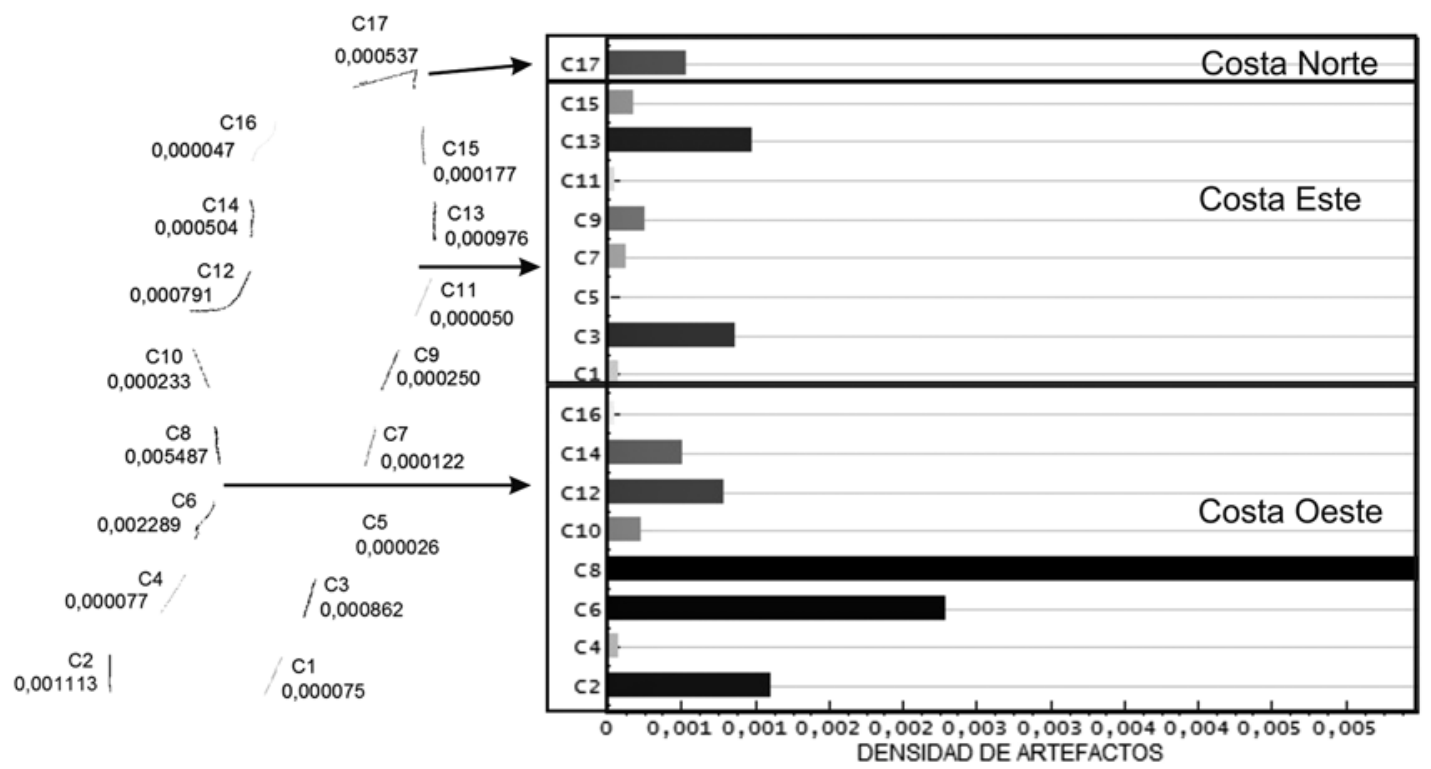

Fig. 6. Densidad de artefactos líticos en la costa.

De esta manera, observamos que existen diferencias entre las unidades analíticas, dado que se encontró mayor densidad en la costa Oeste (costa de la bahía). Por otra parte, las transectas con densidad mayor a 0,0010 se hallan dispersas (Fig. 6 y Fig. 7), dos en Norte de la península (transectas interiores 15 y 17), dos en el centro (transectas de la costa C6 y C8) y una en el sur (transecta C2).
Como se puede observar en la Fig. 8 (que muestra la densidad para cada segmento de transecta) y en la Fig. 9 (que muestra los segmentos de densidades mayores a 0,024 [10 o más artefactos por segmento] y las concentraciones artefactuales [sitios] identificadas fuera de transecta) la densidad de artefactos líticos no es homogénea para cada unidad analítica, evidenciando áreas de mayor densidad
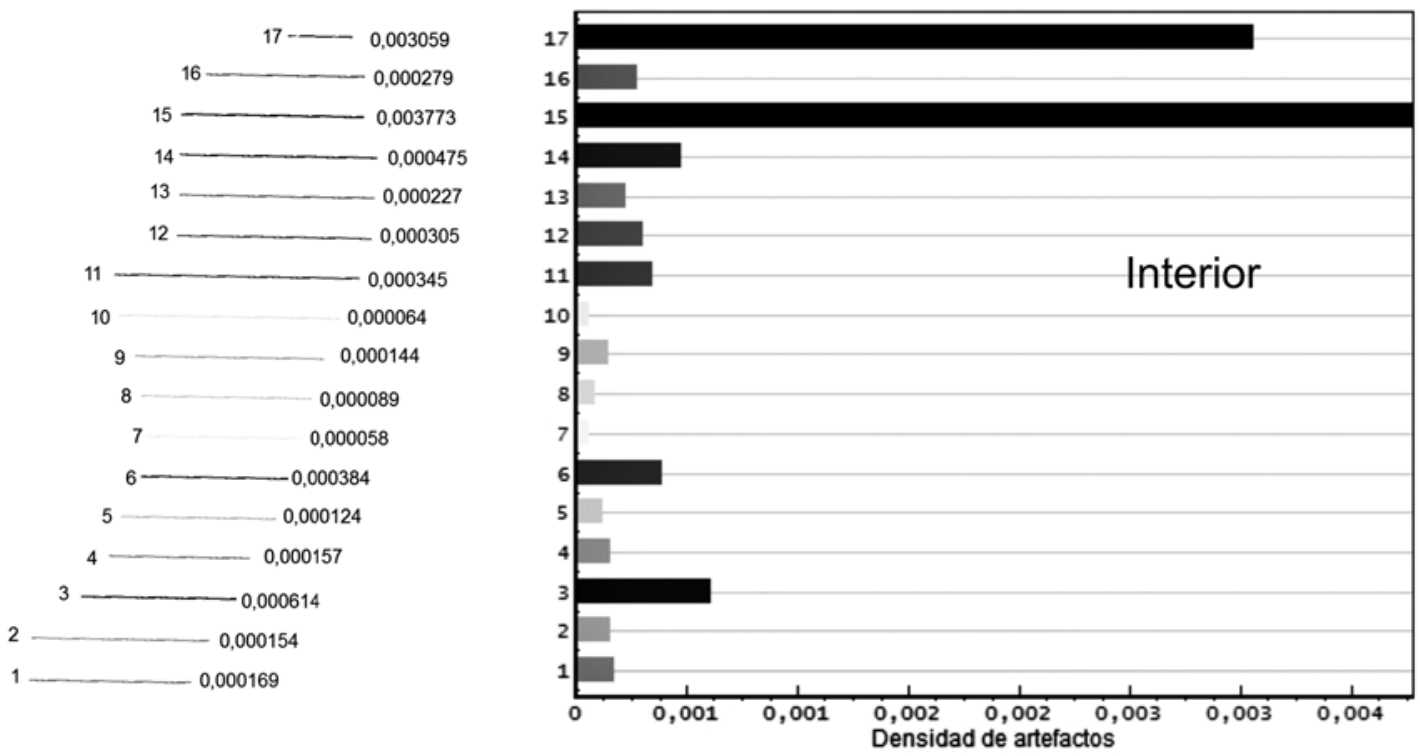

Fig. 7. Densidad de artefactos líticos en el interior. 


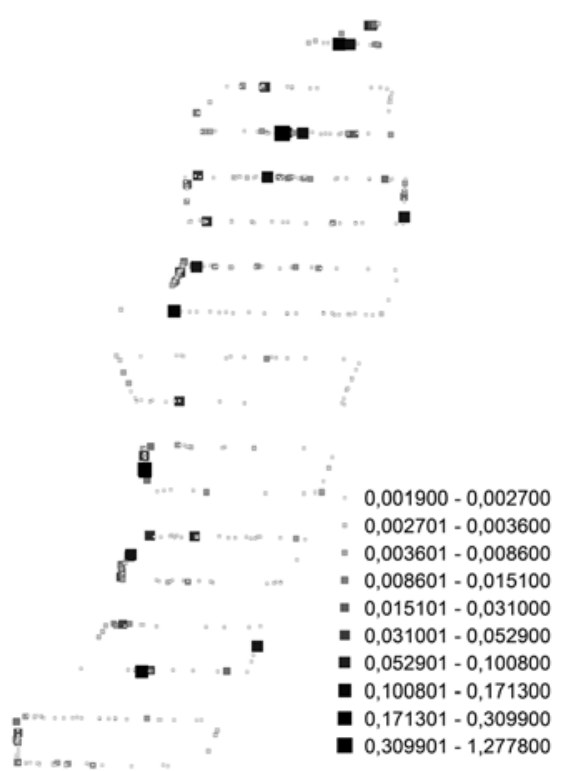

Fig. 8. Densidad dentro de transecta.

artefactual distribuidas de manera discreta pero a lo largo de toda la península.

El modelo de densidad artefactual (Fig. $10 \mathrm{y}$ Fig. 11) muestra los sectores con mayor concentración vinculados a las zonas de acceso y estrangulamiento

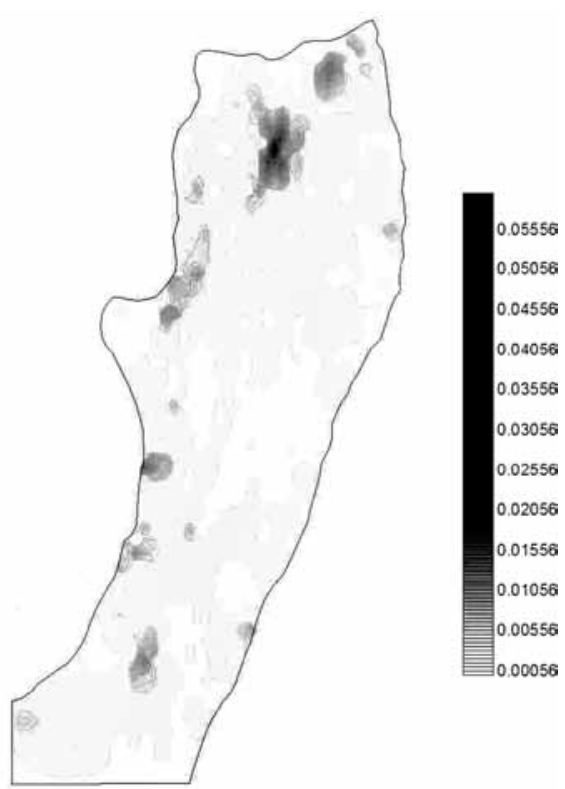

Fig. 10. Modelo de de densidad artefactual.

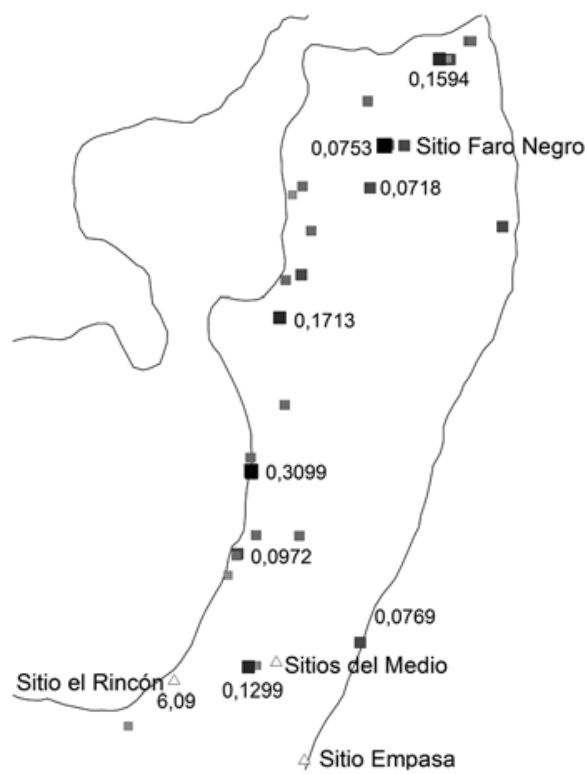

Fig. 9. segmentos con densidad mayor a 0,024 y sitios identificados. (sectores 1 y 5), la costa de la bahía (sector 2) zona de bajos y lagunas sin agua durante el verano (sector 3 ) y la costa norte (sector 4). Este patrón de distribución podría estar relacionado con los recursos, el reparo y/o la geomorfología.

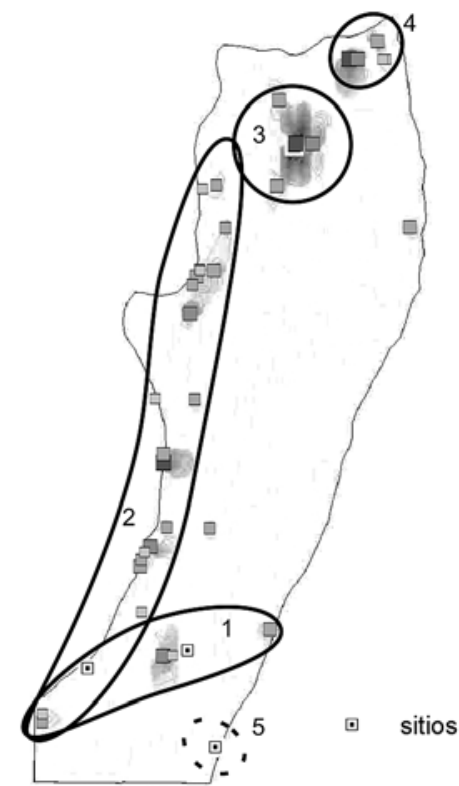

Fig. 11. Áreas de mayor densidad. 


\section{DISCUSIÓN Y CONCLUSIÓN}

La distribución espacial registrada indica tres áreas de alta densidad artefactual, una vinculada a la costa de la bahía, otra en la zona de acceso y estrangulamiento, y la tercera en el extremo norte, con pequeños bajos y lagunas temporarias. Como escribimos arriba, la densidad de artefactos no es homogénea para cada unidad analítica, evidenciando concentraciones en lugares particulares de la península. Se visualiza una diferencia apreciable entre una gran extensión con muy baja densidad artefactual, entre $0,000026 \mathrm{art} / \mathrm{m}^{2}$ y 0,005487 $\mathrm{art} / \mathrm{m}^{2}$, en relación a la registrada en los sectores de mayor concentración, que oscila entre 6,09 en sitio El Rincón (Frank y Skarbun 2007) y 0,002587 $\mathrm{art} / \mathrm{m}^{2}$ (con locus de densidades mayores a 0,99 $\mathrm{art} / \mathrm{m}^{2}$ ) en sitio Del Medio, ambos poseen sectores viables de excavación y se encuentran ubicados en la zona angosta de la península, el primero en la costa de bahía y el segundo en el interior. Por otro lado, las concentraciones del extremo norte poseen una densidad de $1,28 \mathrm{art} / \mathrm{m}^{2}$, cercanas a pequeños bajos y lagunas temporarias $\mathrm{y}$ a las restingas donde pueden obtenerse mejillones. Estos elementos muestran, por un lado, un uso diferencial de los espacios y por otro, un desarrollo de las actividades en sitios acotados, lo que podría estar relacionado con los recursos y la topografía. Con pocas zonas grises, es decir se pasa de concentraciones altas a grandes sectores con muy baja densidad artefactual en pocos metros de recorrido. En forma general podemos decir que las densidades artefactuales obtenidas están dentro de lo esperable para extensiones de baja densidad en este tipo de ambiente. Por ejemplo, zona de El Salitral de Cabo curioso, ubicado $20 \mathrm{Km}$ al norte de Puerto San Julián: 0,00027 a 0,00059 art/ $/ \mathrm{m}^{2}$ (Lanata et al 2004), zona del golfo de San José: 0,000105 art/ $\mathrm{m}^{2}$ (Belardi 2003), costa atlántica de Cabo Blanco: 0,0000739 art $/ \mathrm{m}^{2}$ (Zubimendi 2010).

En cuanto a las densidades propias de los sitios, los antecedentes en la costa de Santa Cruz, vienen mostrando diferencias sitio por sitio, coherente con la variabilidad registrada, en cuanto a función y características contextuales, por lo que consideramos poco significativo hacer comparaciones entre sitios sin considerar otras variables, análisis que excede los objetivos de este artículo
(Belardi 2003; Castro et al. 2003; Caracotche et al. 2008; Zubimendi 2010).

No obstante, si cabe recordar que los estudios en la Costa Norte de Santa Cruz muestran, según las evidencias registradas en los sitios, una intensificación en la explotación de recursos marinos, debidos a una alta disponibilidad del ambiente (Moreno 2003; Castro et al.2003; Zubimendi et al. 2004; 2005). Situación parecida a Monte León donde predomina el consumo de recursos costeros, mamíferos marinos y moluscos (Caracotche et al. 2008). En este sentido, son claras las diferencias con los resultados del sector que estamos presentando, donde la subsistencia parece estar basada principalmente en el guanaco.

En este orden, los datos obtenidos en los sitios en estudio El Rincón y Del Medio, ubicados en la zona de acceso y estrangulamiento de la península, si bien todavía no tenemos valores estadísticos referidos a fauna utilizada, indican en el registro una gran predominancia de restos óseos de guanaco y presencia de artefactos elaborados sobre elementos de esta especie en el primer sitio nombrado, en un contexto de múltiples actividades (Frank y Skarbun 2007). Es clara la ausencia total de pinnípedos y aves marinas. Los moluscos, casi exclusivamente representados por mejillón, se encuentran en cantidades muy bajas, dispersos y sin conformar acumulaciones con características de conchero. Estos datos, se suman a las evidencias distribucionales que estamos presentando que señalan locaciones en lugares particulares del paisaje no necesariamente vinculados a la utilización de recursos marinos. En este orden, la ubicación de campamentos en la entrada de la península, refuerza la hipótesis referida a un control de las manadas de guanacos para utilización y consumo, mediante campamentos en su acceso.

En nuestra agenda quedan muchas tareas a realizar en la Reserva Península de San Julián y las preguntas actuales justifican las investigaciones en curso, estas están referidas a continuar los estudios intrasitios, la excavación de los pequeños sectores estratificados identificados, la obtención de edades radiocarbónicas y las clasificaciones taxonómicas en terreno, entre otras actividades necesarias en este proyecto, en el marco de un conocimiento regional que integre los contenidos arqueológicos producidos merced las investigaciones propias y ajenas referidas a meseta, costa y zonas intermedias de la provincia de Santa Cruz. 


\section{AGRADECIMIENTOS}

Queremos agradecer a Pablo Rastelli, Julián Gauto y Natalia Manessi del Consejo Agrario Provincial, sede San Julián. Particularmente a Natalia Lunazzi, Matías Paunero, Martín Del Giorgio y Lic. Ariel Frank, compañeros del proyecto, quienes participaron activa y animosamente en los trabajos de campo. A Dra. Alicia Castro y Lic. Juan Pablo Martín por su asesoramiento y apoyo. A la comunidad y Municipalidad de Puerto San Julián. Estas investigaciones se realizan en el marco del proyecto: 11/N530. SeCyT. UNLP. Arqueología y Comunicación en la Reserva Península de San Julián, provincia de Santa Cruz.

\section{BIBLIOGRAFÍA}

ARRIGONI, G., M. ANDRIEU y C. BAÑADOS 2008. Arqueología de cazadores-recolectores prehistóricos en la costa central del Golfo San Jorge. Arqueología de la Costa Patagónica. Perspectivas para la conservación. I. Cruz y M. S. Caracotche (Eds.), pp. 91-107. Universidad Nacional de la Patagonia Austral - Subsecretaría de Cultura de la Provincia de Santa Cruz. Río Gallegos.

BELARDI, J.B. 2003. Paisajes Arqueológicos: Un estudio comparativo de diferentes ambientes patagónicos. Facultad de Filosofía y Letras, UBA, Buenos Aires. Tesis Doctoral. MS

BEVAN, A. y J. CONOLLY 2009. Modelling spatial heterogeneity and nonstationarity in artifact-rich landscapes. Journal of Archaeological Science 36: 956-964.

BINFORD, L.R. 1992. Seeing the Present and Interpreting the Past -and Keeping Things Straight. Space, Time, and Archaeological Landscapes. J. Rossignol y J. Wandsnider (Ed). New York, Plenum.

BORRERO, L.A., J.L. LANATA y B.N. VENTURA 1992. Distribuciones de hallazgos aislados en Piedra del Águila. Análisis espacial en la arqueología patagónica. Editado por L. A. Borrero y J. L. Lanata. Pp. 9-20. Ediciones Ayllu, Buenos Aires.

BORRERO, L.A., N.V. FRANCO, R. BARBERENA, F. BORELLA, P. CAMPAN, F. CARBALLO MARINA, I. CRUZ, C.M. FAVIER DUBOIS, R. GUICHON, G. L'HEUREUX, M.V. MANCINI, L. MANZI y F. MARTIN 2008. Arqueología de Cabo Vírgenes y Cañadón Gap. Arqueología de la Costa Patagónica. Perspectivas para su conservación. I. Cruz y M. S. Caracotche (Ed).
Pp. 212-228. Universidad Nacional de la Patagonia Austral - Subsecretaría de Cultura de la Provincia de Santa Cruz. Río Gallegos.

CARACOTCHE, M.S., I. CRUZ, S. ESPINOSA, F. CARBALLO MARINA y J.B. BELARDI 2005. Rescate arqueológico en el Parque Nacional Monte León (Santa Cruz, Argentina). Magallania 33(2): 143-163.

CARACOTCHE, M.S., M.F. CARBALLO, J.B. BELARDI, I. CRUZ y S.L. ESPINOSA 2008. El registro arqueológico del Parque Nacional Monte León (Santa Cruz): Un enfoque desde la conservación. Arqueología de la Costa Patagónica. Perspectivas para su conservación. I. Cruz y M. S. Caracotche (Ed). Pp. 147-159. Universidad Nacional de la Patagonia Austral - Subsecretaría de Cultura de la Provincia de Santa Cruz. Río Gallegos.

CASTRO, A. y MORENO J.E. 1998. Un sitio del Holoceno medio en la costa norte de Santa Cruz. Revista $\mathrm{Pa}$ limpsesto: 135-137.

CASTRO, A. y MORENO J.E. 2000. Noticia sobre enterratorios humanos en la Costa Norte de Santa Cruz. Anales del Instituto de La Patagonia. Serie Ciencias Humanas. Vol. 28:225-233.

CASTRO, A.S., J.E. MORENO, M.A. ANDOLFO, M.A. ZUBIMENDI, R. GIMENEZ, L. MAZZITELLI y P. AMBRÚSTOLO 2003. Análisis distribucionales en la costa de Santa Cruz (Patagonia Argentina): alcances y resultados. Anales del Instituto de la Patagonia. Serie Ciencias Humanas 31: 69-94.

CASTRO, A.S., J.E. MORENO, M.A. ANDOLFO, M.A. ZUBIMENDI, B. VIDELA, P. AMBRUSTOLO y L. MAZZITELLI 2008. Cazadores recolectores costeros: interpretaciones desde el registro arqueológico de la costa norte de Santa Cruz. Arqueología de la Costa Patagónica. Perspectivas para su conservación. I. Cruz y M. S. Caracotche (Ed). Pp. 128-144. Universidad Nacional de la Patagonia Austral - Subsecretaría de Cultura de la Provincia de Santa Cruz. Río Gallegos.

CODIGNOTTO, J.O. 1990. Evolución del Cuaternario alto del sector de costas y plataforma submarina entre Coig, Santa Cruz y Punta María, Tierra del Fuego. Revista de la Asociación Geológica Argentina 45 (1-2): 9-16.

CODIGNOTTO, J.O. 1996. Cuaternario y Dinamica Marina. XIII Congreso Geológico Argentino y II Congreso de Hidrocarburos. Geología y Recursos Minerales de la Plataforma Continental Argentina. V.A. Ramos y M.A. Turic (Eds.). Relatorio 2: 17-287. Buenos Aires.

DARWIN, C.H. 1886. A naturalist's voyage: Journal of researches during the voyage of H.M.S. "Beagle" round the world. London. J. Murria. 
DUNNELL, R.C. 1992. Cap. 2: The Notion Site. Space, Time, and Archaeological Landscapes. J. Rossignol y L. Wandsnider (Ed). Pp. 21-41. New York, Plenum.

FERUGLIO, E. 1950. Descripción Geológica de la Patagonia. YPF, 3 tomos. Buenos Aires.

FOLEY, R. 1981. A model of regional Archaeological Structure. Proccedings of the Prehistoric Society 47: 1-17.

FRANK, A.D. y F. SKARBUN 2008. Análisis de la distribución de restos arqueológicos en el sitio El Rincón, localidad arqueológica Reserva Península de San Julián. Arqueología de Patagonia: una mirada desde el último confín. Pp. 999-1010. Editorial Utopías. Ushuaia.

IZETA, A.D. 2008. Análisis espacial del descarte de camélidos en una unidad doméstica formativa. Loma alta núcleo "e" (Catamarca, Argentina). Archaeobios 2: 1-10.

LANATA, J. L., S. BUSCAGLIA, M. CARDILLO, S. FRETE, M. MARSCHOFF, A. GARCÍA, G. HERBST, V. NUVIALA y C. OTAOLA. 2004. Cazadores recolectores en Puerto San Julián, Santa Cruz. Primeros resultados. Contra viento y marea. Arqueología de Patagonia. Pp. 745754. Instituto Nacional de Antropología Pensamiento Latinoamericano. Buenos Aires.

MANESSI, N. 2007. Proyecto: Refacción del casco histórico y construcción de una vivienda en la Reserva Provincial Península de San Julián, Ley provincial $N^{\circ}$ $1821 / 86$. MS.

MANSUR, M.E. 2008. Arqueología de la zona de Punta Bustamante (Provincia de Santa Cruz, Argentina). Arqueología de la Costa Patagónica. Perspectivas para su conservación. I. Cruz y M. S. Caracotche (Ed). Pp. 161-173. Universidad Nacional de la Patagonia Austral
- Subsecretaría de Cultura de la Provincia de Santa Cruz. Río Gallegos.

MENA F.L. 1988-89. Hacia un panorama del registro arqueológico: Promesas y frustraciones. Arqueología Contemporánea Vol. 2 № 2: 31-52. Buenos Aires.

MORENO, J.E. 2003. El uso indígena de la costa patagónica central en el Período Tardío. Facultad de Ciencias Naturales y Museo. Universidad Nacional de La Plata., La Plata. Tesis Doctoral. MS.

PANZA, J.L.E, M.V. IRIGOYEN 1994. Hoja 4969-IV: Puerto San Julián, provincia de Santa Cruz. Dirección Nacional del Servicio Geológico. Boletín № 211. Buenos Aires.

PEREYRA, F.X., L. FAUQUÉ y GONZÁLEZ DÍAZ, E.F. 2002. Geomorfología. Geología y Recursos Naturales de Santa Cruz. M.J. Haller (Edit.). Relatorio del XV Congreso Geológico Argentino. 1-21: 325-352. Buenos Aires.

ROSSIGNOL, J. 1992. Chapter 1: Concepts, Methods, and Theory Building. Landscape Approach. Space, Time, and Archaeological Landscapes. J. Rossignol y L. Wandsnider (Ed). New York, Plenum.

ZUBIMENDI, M.A. 2010. Estrategias de uso del espacio por grupos cazadores recolectores en la Costa Norte de Santa Cruz y su interior inmediato. Facultad de Ciencias Naturales y Museo. Universidad Nacional de La Plata. La Plata. Tesis Doctoral. MS

ZUBIMENDI, M.A., A.S. CASTRO Y J.E. MORENO 2004. Una aproximación hacia la definición de modelos de uso de la Costa Norte de Santa Cruz. Magallania 32: 85-98.

ZUBIMENDI, M.A., A.S. CASTRO Y J.E. MORENO 2005. El consumo de moluscos en la Costa Norte de Santa Cruz. Intersecciones en Antropología 6: 121-137. Olavarría. 